Wasserstoffsuperoxyd geführt, wobei eine dunkelblane läroung entstand.

Eine Iintersuchung auf andere farbsalzbildende Metalle wie Eisen oder Kupler verlief negativ.

Die Anwesenheit von Chrom im Veraschungsriickstand läßt darauf schließen, daß für die Gummierungen der verschiedenen Klebestreifen Lederleime oder Gemische von Haut- und Lederleimen verwendet worden waren. Diese enthalten, cla sie aus entgerbten Chromlerlerabfällen hergestellt werden, mehr oder weniger große Reste von Chromsalzen, die mit hoher Wahrscheinlichkeit für die 1)unkelfärbungen verantwortlich zu machen sind.

Wie aus der Technik der chemischen Beizen bekannt ist, benutzt man Chromsalze als Nachbeizen für gerbsäurehaltige oder mit gerbsäurehaltigen Vorbeizen behandelte Furniere. Hierdurch wird eine irreversible Tunkelfärbung hervorgerufen, deren Intensität von der Konzentration der Reaktionspartner abhängt. Die Farbreaktion verläuft jedoch langsam, wird aber z. B. durch Ammoniak beschleunigt und vertieft. Dies scheint auch der Grund dafür zu sein, dal die anfangs kaum sichtbaren Abzeichnumgen erst beim Auftragen von Ammoniakbeizen deutlicher zum Vorschein kommen. Infolge ihrer Tiefenwirkung sind diese Verfärbungen mit mechanischen Mitteln kaum zu entfernen.

\section{Zusammenfassung}

l) ie Markicrungen von lílebestreifen auf Decklurnieren beruhen, abgesehen von den rein mechanischen Stauchungen durch zu dicke Papiere, fast immer auf chemischen Reaktionen. Wie bei allen Verfärbungserscheinungen von Hölzen sind ihre Trsachen häufig unklar.

Zwet solcher Verfärbungen, die zeitweise die Mobblindustrie sehr beunruhigten, konnten jedoch aufgeklärt werrlen.
Bei den Hellverfärbungen handelte es sich um Bleichungen der Furnieroberfäche durch Natriumdithionit, das dem Gummierleim vermutlich in zu großen Mengen zugesetzt worden war

Die Dunkelverfärbungen waren auf die Verwendung von T.ederleimen oder Lederleimzusätzen für die Gummierungren der Klebestreifen zurückzulühren, die beträchtliche Mengen an Chromverbindungen enthielten. 1)iese hatten mit den Gerbstoffen verschieclener Holzer clunkle Reaktionsprodukte gebildet, deren Farbtiele durch ammoniakalische Beizen verstärkt murde.

\section{Summary}

Apart from purely mechanical upsets ouing to too thich paper, the marking of paper tapes on surfacing rencers is almost always based on chemical reactions. As in all discolorations of wood, its cause is frequently unkmpn. Two of these discolorations, which had formerly troubled the furviture industry, can now be explained.

Light discolorations are caused by bleachinir the reners by: sodiumdithionite which had probably been added to the glue in too large quantities.

Darh discolorations are due to the use of leather glues or admixtures of leather glue to the gum of the tapes which contained considerable quantities of chromic compounds. Touether with the tannins of the different wood species the compounds formed dark reaction products, whose colour was deepened by staining with anmoniat solutions.

\section{Schrifttum}

1 Kollmann, $F$, R. Keylwerthu, Il Kübler: Ver. fürbungen des Vollholzes und der Furniere bei der künstlichen Holetrocknung. Holz als Roh-und Terkstoff Bal.9. (1951) S. $392 / 391$ - 2. Sandermann. TY.. u. M. Lïtlogons: Lntersubungen äber Verfärbugen von Hölzern. Ilsh als Hoh. und Werkstoff Pd. 11 (1953) S. $435 / 40$.

\title{
Nachtrag zur Würdigung für Ernst Gieseking
}

(Heft 5, 1958)

Wie mir mitareteilt warde. erfolerte die Grïustung der

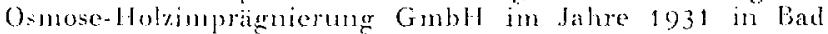

Kissingen seitens der Herren Ing. Carl Schmittulz um 1)r. Ing. Lrast Gieseking.
F. Kollonem Münchens.

\section{Kleine Mitteilungen}

\section{Entwicklung und Herstellung von Holzspanplatten}

Bericlit äber die silzung des gleichnamigen Arboilsauschusses der Deutschen Gesellschaft fiir Holzorshung aus Anlaß seines $10 \mathrm{j}$ abrigen Beslehens a m lo. Junj 1958 in Braunschweig.

Auf der diesjahrigen Farhtagung des Arbeitsausschusses nafer sich an traditionellen Tagungsort Braunschweig mehr als 1 og Vituglieder und Gäste, unter denen sich Teilnehmer aus don l indern Frankreich, England, Jom egen, Östereich und dex Scluweiz befanden. Zu Berinn berichtete ler Obmann des Ausshusses, Dr. Ing. W. Klauditz (Braunschweig), kur. iber die Tatiglieit des Ausschusses wihrend der letzlen heiden Jahre und hob dabei herror, daB rom Institut fiar Holz forschung a. d. Techn. Hochschule Bramschweig Erhebungen iiber Produkion und Rohbolzversorgung der Holaspanplationindustrie durchgeführt worden seien, um oine möglichst stefige und ibbersichthohe Heiterentwicklung dieses "irhtigen Fndustriezweiges zu sichern; die Ligebusse sind in 2 lierichten des Instiluts für Holzforschung a. d. Techn. IIochschule Braunschweig inzwischen bekanntiregeben*. Als vichlige schriftumsgrundlage hat auf Amregung des Aus. schusses das Institut für Leichtbau d. Techn. Hochschule Braunschwcig unter Leitung von Prof. Dr. Winter einen gebundenen Literaturbericht herausgegeben. Weiterhin wurden kurz die Erobnisse der FAO-Konlerenz über I Holzfascr- und Holzspanplatten in Genf (1957) sowie der 4. FAOKonferen\% fiir [lolztechnologie in Malrid (1958) gestreift, auf der cine Arbeitsgruppe für die Prüfung von Holzspanplatten gebildet wurde (J.Collardet-Frankreich, A. Gratzl-Öster- reich, H. kiihne-Solweiz, B. Thunell-Sohwolen. k. kevlwerth und W. Klauditz-Denischland) - Ferner arab Dr. W. Klauditz bekannt, daB im Arboitsausschul der Doutschen Gesellschaft für Holzforschung ,Entwicklung und Herstellung von Holzspamplation"s in Znliunft auch Prïfunersfragen behandelt werden, fiir die fribher cin besonderer Ausschul gegrindet worden sei; beide werden nummehx ver einigt ihre latigrlieit fortsetzen.

Ansehliebend schilderle Dr. IV.KJ andilz den Verde gang des Arboitsansschusses, Holzspanplaten:", dessen Grimdung 1047 an die trbeit des Tereins fin "Terhmischo Holffragen in Bramschweig zurücdigelat. Tn verhälnismaibig linzer Zeit war es durch Zusammenarbeit aller interessierten Stellen aus Forschmor, Forstoirlsthate, Holzwirtselaft und Industrie möglich, der Holzspanplattenherslellung eine gut gesicherte wissenschafiliche und technische Grundlage $\%$

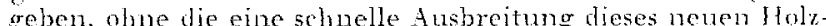
industriezweiges nicht zustande gekommen wäe. Als hus sprachebasis fior alle wissenschaftichen umd rechmischon Probleme auf den Holrspanplatengebiet hat sich der Arbeils ausschuld mit seinen Vortragsveranstaltungen und fachlichen Sitzungen bestens bewähre. Dr. WV. Klaudity umrily dan die Futwiclinung der Holzspanplattenindustrie in Dentschland, , die als selbststandiger Zweig innerhalb der Holzindustrie mit noch groben techmischen Möglichkeiten aufzufassen is " Zilal aus dem Jahre 1951). Wichtige Stationen der tech nishen Hntwicklung waren: der Bau geeigneler Zerspanungs maschinen, Beleimungsmaschinen, Streumaschinen usw. welche die Errichtung rröBerer selbständirer Werlie ab 1951 ermöglichten (Norddeutsche Holzwerkstoff-Gesellschaft mbH. 